\title{
Insatisfação corporal de adolescentes escolares
}

$\mathrm{Na}$ adolescência o corpo passa por mudanças físicas, psicológicas, biológicas com mudanças sociais resultando em conceitos de si mesmo e de sua composição corporal. A insatisfação e a desvalorização de si na questão físico traz distúrbios para a vida de uma pessoa, por conta da adoção de atitudes que visam a mudança do estado. $O$ objetivo desse estudo foi analisar metodologicamente as pesquisas publicadas sobre a avaliação da satisfação corporal de adolescentes escolares. Trata-se de uma revisão sistemática da literatura, cuja metodologia se baseou em investigações anteriores e em recomendações sobre o tema, realizaram-se buscas nas bases Scielo e no Lilacs, utilizando os descritores imagem corporal e adolescentes, combinados a partir do operador booleano 'OR', totalizando assim 5 artigos como amostra final da presente pesquisa. Foi possível concluir que houve maiores índices de insatisfação corporal em adolescentes do sexo feminino por estarem acima do peso, o que pode estar relacionado a não realização de atividades físicas e não adoção de hábitos saudáveis, os dados do estudo justificam a importância de tratar e prevenir os distúrbios de imagem corporal, para ajudar a alcançar os padrões alimentares mais saudáveis.

Palavras-chave: Imagem corporal; Adolescentes; Insatisfação Corporal.

\section{Body dissatisfaction among school adolescents}

In adolescence, the body undergoes physical, psychological, biological changes with social changes resulting in concepts of itself and its body composition Dissatisfaction and devaluation of oneself in the physical issue brings disturbances to a person's life, due to the adoption of attitudes aimed at changing the state. The aim of this study was to methodologically analyze the published research on the assessment of body satisfaction in school adolescents. This is a systematic review of the literature, the methodology of which was based on previous investigations and recommendations on the topic, searches were carried out in the Scielo and Lilacs databases, using the descriptors body image and adolescents, combined from the Boolean operator 'OR', thus totaling 5 articles as the final sample of this research. It was possible to conclude that there were higher rates of body dissatisfaction in female adolescents for being overweight, which may be related to not performing physical activities and not adopting healthy habits, the study data justify the importance of treating and preventing body image disorders, to help achieve healthier eating patterns.

Keywords: Body image; Teens; Body dissatisfaction.

\section{Topic: Educação Física}

Reviewed anonymously in the process of blind peer.
Received: 19/09/2019

Approved: $24 / 01 / 2020$
Marcos Antonio Araújo Bezerra (iD)

Centro Universitário Dr. Leão Sampaio, Brasil

http://lattes.cnpq.br/4643352879633283

http://orcid.org/0000-0002-3385-4024

marcosbezerra@univs.edu.br

Gisele Gonçalves de Barros

Centro Universitário Dr. Leão Sampaio, Brasil

http://lattes.cnpq.br/5978402564240491

http://orcid.org/0000-0003-4442-2005

giselebarrosed@gmail.com

Gabriela Gomes de Oliveira Bezerra (it)

Centro Universitário Dr. Leão Sampaio

http://lattes.cnpq.br/6330341118187369

http://orcid.org/0000-0002-9724-708X

gabriela.o.bezerra@gmail.com
José de Caldas Simões Neto (DD

Centro Universitário Dr. Leão Sampaio, Brasil

http://lattes.cnpq.br/0470733825644726

http://orcid.org/0000-0003-1036-2315

josecaldas@leaosampaio.edu.br

Lara Belmudes Bottcher (iD

Centro Universitário Dr. Leão Sampaio

http://lattes.cnpq.br/4112151773664370

http://orcid.org/0000-0002-5504-4102

larabottcher@leaosampaio.edu.br

Cícero Cleber Brito Pereira (id

Centro Universitário Vale do salgado, Brasil

http://lattes.cnpq.br/6554764959422181

http://orcid.org/0000-0002-9402-9810

cicerocleber@univs.edu.br

\section{Referencing this:}

BEZERRA, M. A. A.; BARROS, G. G.; BEZERRA, G. G. O.; SIMÕES NETO, J. C.; BOTTCHER, L. B.; PEREIRA, C. C. B.. Insatisfação corporal de adolescentes escolares. Health of Humans, v.2, n.1, p.17-23, 2019. DOI: http://doi.org/10.6008/CBPC2674-6506.2020.001.0003 


\section{INTRODUÇÃO}

O corpo passa por mudanças físicas, psicológicas, biológicas, iniciando a adolescência, por volta dos 10 anos de idade, período esse de muitas transformações nas percepções de cada indivíduo, resultando em conceitos de si mesmo, de sua composição corporal, de como se enxerga principalmente se comparando com outras pessoas (AINETT, 2017; PETROSKI, 2012).

Taborda et al. (2014), relatam quando estudam adolescentes com idades entre 13 e 18 anos, que as transformações biológicas representam eventos importantes nos processos de formação do indivíduo e desenvolvimento humano, pois essas transformações ocorrem a nível físico em razão das alterações hormonais como também alterações e no cérebro ganhando proximidade com a mente adulta.

O peso acima do padrão de beleza gera preocupações e pressões socais durante toda a vida, na adolescência essas influencias só aumentam (MARTINS et al., 2015). As pesquisas de monitoramento da saúde, mostram que nos últimos anos há uma tendência no aumento de peso e crescimento da obesidade, e adolescentes demostram preocupação com o corpo quando $80 \%$ indicam importante ou muito importante a imagem corporal que apresentação, entretanto esses indivíduos não procuram as formas e os meios saudáveis para atingir o objetivo é mudar a sua realidade (MARTINI et al., 2020).

De acordo com as afirmações de Guimarães et al. (2020), onde falam sobre a percepção do corpo, a imagem real e os desejos futuros, podemos entender os conceitos de insatisfação ou descontentamento com o corpo ou com imagem corporal quando se é distorcido o que se enxerga do seu corpo naquele momento tendo parâmetro as comparações com os objetivos que tanto deseja e almeja, e dessa forma passa a enxergar a imagem corporal atual de forma negativa.

A insatisfação e a desvalorização de si na questão físico traz distúrbios para a vida de uma pessoa, por conta da adoção de atitudes que visam a mudança do estado, podemos citar a alimentação como umas das primeiras atingidas, quando se pratica dietas restritivas (VIANA, 2014). Neste sentido o processo de perca de peso ocorre uma baixa de sua autoestima, procurando acentuar os resultados pode ocorrer o uso de medicamentos sem prescrição médica assim como adotar práticas de exercícios com execuções incorretas, resultando de forma global distúrbios psicológicos e sociais. Portanto gera-se prejuízos não só físicos como psíquicos (IEPSEN et al., 2014).

Jovens estudantes podem sofrer frequentemente com a insatisfação corporal. Na zona urbana do município de Irati estado do Paraná, os alunos quando estudados, demonstraram em sua maioria (70,9\%) para insatisfação o que enxergam de seu corpo e dentre elas a grande maioria $(75,5 \%)$ sentem vontade de reduzir o peso atual (SANTOS et al., 2014).

Aliás esse desejo está sempre presente na maioria dos indivíduos, independentemente de sua classificação no IMC (Índice de Massa Corporal) (LIMA et al., 2018). Nessa população estudada por Santos e colaboradores foi encontrado uma insuficiência ativa de $36,5 \%$ junto de um baixo peso de $42,4 \%$. Neste estudo, apesar da alta prevalência, não houve relação entre a atividade física com a auto avaliação corporal (SANTOS et al., 2014). 
O interesse em estudar este tema surgiu e foi aumentando de proporções durante atividades no campo de estágio em escolas, os relatos dos alunos quando faziam comentários sobre si, levantavam suspeitas em direção a insatisfação com a imagem corporal. A relevância do estudo se dá quando há uma necessidade de abordar esse tem, para que possa despertar os profissionais responsáveis para a criação de novas estratégias, projetos e intervenções para tratar deste tema com os alunos, gerando informação e saúde. Portanto o objetivo desse estudo foi analisar metodologicamente as pesquisas publicadas sobre a avaliação da satisfação corporal de adolescentes.

\section{METODOLOGIA}

Trata-se uma revisão sistemática da literatura, cuja metodologia se baseou em investigações anteriores e em recomendações sobre o tema, realizaram-se buscas na base Scielo e no Lilacs, utilizando os descritores imagem corporal e adolescentes, combinados a partir do operador booleano 'OR'. Logo, em seguidas foram aplicados os seguintes critérios de inclusão: ser original com resumo disponível; ter sido realizado com adolescentes 'saudáveis'; ter sido publicado nos últimos seis anos (2014 a 2020). Foram excluídos artigos de revisão sistemática, bibliográfica, meta-análises estudos duplicados e estudos em língua estrangeira. Foram encontrados a partir das bases de dados eletrônicas $(n=3560)$ artigos, sendo $(n=1746)$ artigos na plataforma SCIELO, e (n=1814) artigos na plataforma LILACS. Quando aplicado o critério de inclusão restaram 21 artigos, por fim foram excluídos 16 artigos de acordo com os critérios de exclusão, totalizando assim 5 artigos como amostra final da presente pesquisa (Fluxograma 1).

\begin{tabular}{|c|c|}
\hline \multicolumn{2}{|c|}{ IDENTIFICAÇÃo } \\
\hline $\begin{array}{l}\text { Registros identificados através das bases de dados } \\
\text { (Scielo) } \\
\underline{\mathbf{1 . 7 4 6} \text { artigos }}\end{array}$ & $\begin{array}{l}\begin{array}{l}\text { Registros identificados através das bases de dados } \\
\text { (Lilacs) }\end{array} \\
\underline{1.814 \text { artigos }}\end{array}$ \\
\hline \multicolumn{2}{|c|}{ SELEÇÃO } \\
\hline \multicolumn{2}{|c|}{$\begin{array}{l}\text { Aplicados os critérios de inclusão (ser original com resumo disponível; ter sido realizado com adolescentes } \\
\text { "saudáveis"; ter sido publicado nos últimos seis anos } 2014 \text { a 2020) }\end{array}$} \\
\hline \multicolumn{2}{|c|}{$\frac{V}{\text { ELEGIBILIDADE }}$} \\
\hline \multicolumn{2}{|c|}{$\begin{array}{c}\text { Critérios de exclusão. (Artigos de revisão sistemática, bibliográfica, meta-análises estudos duplicados e estudos em } \\
\text { língua estrangeira.) }\end{array}$} \\
\hline \multicolumn{2}{|c|}{ INCLUSÃO } \\
\hline Selecionados & ião nordeste. \\
\hline
\end{tabular}

Fluxograma 1: Seleção dos estudos. 
Tabela 2: Síntese dos estudos das prevalências de insatisfação corporal de escolares.

\begin{tabular}{|c|c|c|c|c|c|c|c|}
\hline Autores/Ano & Local & $\begin{array}{ll}\text { Tipo de } \\
\text { estudo }\end{array}$ & Seleção & Amostra & $\begin{array}{l}\text { Faixa } \\
\text { etária }\end{array}$ & Instrumentos & $\begin{array}{l}\text { Insatisfação } \\
\text { corporal }\end{array}$ \\
\hline $\begin{array}{l}\text { Bandeira et al. } \\
(2016)\end{array}$ & Fortaleza & Transversal & Conglomerado & 300 & $\begin{array}{l}17-24 \\
\text { anos }\end{array}$ & $\mathrm{BSQ}$ & $47,0 \%$ \\
\hline Oliveira (2017) & Bahia & Transversal & Conglomerado & 51 & $\begin{array}{l}10 \text { a } 18 \\
\text { anos }\end{array}$ & $\mathrm{BSQ}$ & $23,56 \%$ \\
\hline $\begin{array}{l}\text { Guimarães et al. } \\
(2020)\end{array}$ & Bahia & Transversal & Conglomerado & 1.582 & $\begin{array}{l}15 \text { a } 24 \\
\text { anos }\end{array}$ & $\begin{array}{l}\text { Escala de silhuetas } \\
\text { de Stunkard }\end{array}$ & $79,5 \%$ \\
\hline Alves et al. (2017) & Fortaleza & Transversal & Conglomerado & 393 & $\begin{array}{l}14 \text { a } 18 \\
\text { anos }\end{array}$ & $\begin{array}{l}\text { Escala de silhuetas } \\
\text { de Stunkard }\end{array}$ & $65,4 \%$ \\
\hline $\begin{array}{l}\text { Ribeiro-Silva et } \\
\text { al. (2018) }\end{array}$ & Salvador & Transversal & Conglomerado & 1.496 & $\begin{array}{l}14 \text { a } 18 \\
\text { anos }\end{array}$ & QIC & $19,5 \%$ \\
\hline
\end{tabular}

Legenda: BSQ: Body shape questionnaire Body shape questionnaire; QIC: Questionário de imagem corporal.

Este estudo teve como objetivo revisar a literatura sobre a insatisfação corporal em adolescentes escolares. Na Tabela 1, apresenta-se a síntese dos resultados de estudos efetuados em várias cidades do Brasil, no período de 2015 a 2019, de forma a contribuir com informações relevantes ao tema. A maior parte desses trabalhos foi realizada nas Regiões Nordeste e Sudeste. Trata-se dos estudos de delineamento transversal, realizados com amostras que variaram de 91 a 74.589 adolescentes com idade entre 10 e 19 anos.

\section{DISCUSSÃO TEÓRICA}

O estudo de Bandeira et al. (2016) teve como objetivo avaliar a imagem corporal e identificar os mais diversos tipos de distorção da imagem corporal, foi realizado na cidade de fortaleza, com 300 universitárias do curso de Nutrição, utilizou-se como instrumento de pesquisa o Body shape Questionnarie (BSQ), instrumento este que avalia o grau de insatisfação com a imagem corporal, além de coletar peso e estatura das alunas para que seja feito o cálculo do IMC. Encontrou-se os seguintes achados, a maioria das estavam eutróficas (75,3\%), uma média geral de IMC de $22,90 \mathrm{~kg} / \mathrm{m}^{2}$ que é classificado como adequado para corpo e peso ideal. O BSQ mostrou que a média do escore foi de 81,43 pontos, sendo assim classificado com insatisfação leve.

Das universitárias estudadas $47 \%$ relataram insatisfação corporal em algum grau, Bandeira et al. concluíram que seus achados corroboram com os trabalhos anteriores, quando afirmar que distúrbios na autoimagem podem ser encontrados em indivíduos eutróficos que não possuem má alimentação, se enxergando com o peso fora do real. Os autores destacaram que o fato da amostra serem estudantes de nutrição, se torna mais expressivo, considerando a função profissional que iram desempenhar.

O estudo de Oliveira (2017) teve como objetivo identificar uma percepção de adolescentes sobre autoimagem corporal, realizado na cidade de Guanambi, na Bahia, com 143 escolares do sexo feminino, faixa etária de 10 a 18 anos, utiliza-se apenas o Body shape Questionnarie (BSQ) como ferramenta para coleta de dados e se encontrou os seguintes itens, $84,31 \%$ cursavam o 60 ano do ensino fundamental e $68,63 \%$ se declaração não católicas.

Existiu predominância positiva em todos os fatores. Na dimensão 'auto percepção da forma do corpo' por exemplo, $76,47 \%$ responderam nunca/raramente/às vezes para se estavam tão preocupadas com seu 
físico a ponto de sentirem que deveriam fazer dieta. Na dimensão 'percepção da imagem corporal comparativa' por exemplo, $74,51 \%$ responderam afirmaram nunca/raramente/às vezes para se olham o físico de outras mulheres e, se comparando, se sentiram em inferiores.

$\mathrm{Na}$ dimensão 'atitude em relação à alteração da imagem corporal', 84,31\% afirmaram nunca/raramente/às vezes que por se sentir mal com o corpo, deixaram de participar de eventos sociais (como, por exemplo, festas). Na dimensão 'grave alteração na percepção corporal', perguntadas sobre a corrida se a evitaram porque seu corpo poderia balançar, 92,16\% responderam nunca/raramente/às vezes.

O tempo e o período letivo foram pontos limitantes desta pesquisa, o que impossibilitou a coleta de dados antropométricos e são característicos nesse tipo de estudo. Temos como conclusão os pontos como a percepção das alunas a sua autoimagem, conhecimento do estilo e hábito de vida, vem a contribuir com a comunidade por se tratar de aspecto, dentro da Saúde Pública, importante.

O estudo de Guimaraes et al. (2020), teve como objetivo analisar a associação entre o consumo excessivo de álcool e a insatisfação com a imagem corporal de adolescentes e jovens que residem em na quarta cidade baiana mais populosa (Camaçari), com 1.582 escolares, predominaram as mulheres $(57,4 \%)$, faixa etária de 15 a 19 anos (56,8\%) e não brancas (89,9\%).

Utilizou-se como instrumento de pesquisa o AUDIT (Teste de Identificação de Desordem de Uso de Álcool) desenvolvido pela OMS para o consumo excessivo de álcool. Uma escala de figura de silhueta para uma insatisfação com a imagem corporal. Encontrou-se os seguintes resultados: grau elevado de insatisfação corporal com porcentagem de 79,5\% dos participantes e uma excessiva ingesta de bebida alcoólica (21,9\%). Tendo uma conclusão de que as hipóteses sendo refutadas pois a o consumo de álcool em excessivo está relacionada a questões religiosas, composição familiar, a idade, estado civil e desemprego.

O estudo de Alves et al. (2017) teve como objetivo: investigar a prevalência de insatisfação com a imagem corporal em mulheres com idade entre 14 e 18 anos e sua associação com o estado nutricional, idade e atividade física, sua amostra final foi de 393 escolares militares. Para tanto, utilizou-se o instrumento escala de silhuetas de Stunkard para a percepção da insatisfação corporal. O estado nutricional foi obtido pela aferição do peso e estatura e após sendo calculado o índice de massa corporal (IMC). A variável relacionada a faixa etária e a atividade física foi obtida por meio de questionário, onde a estudando relatou as duas informações.

Encontrou-se os seguintes achados: $62,6 \%$ das meninas assinalaram praticar atividade física, $63,4 \%$ indicaram estar com peso adequado e 65,4\% alegaram insatisfação com a imagem corporal, tendo uma conclusão que os resultados obtidos são sustentação aos estudos anteriores quando a maiorias das mulheres estudadas estão insatisfeitas com seu corpo, mesmo praticando atividades físicas e estando com o peso dentro do considerado ideal. A variável idade e a pratica de atividade física tiveram associação com imagem corporal.

Ribeiro-Silva et al. (2017) tiveram como objetivo relacionar a insatisfação com a imagem corporal junto dos padrões de alimentação com base no estado antropométrico em estudantes de ambos os sexos, com amostra final de 1.496 alunos de Salvador, Bahia. Utilizou-se como instrumentos o questionário de 
frequência alimentar (QFA), o questionário de imagem corporal (QIC), balança digital e estadiômetro portátil e registros escolares.

Encontrou-se os seguintes achados: alunas do sexo feminino foram maioria $(57,1 \%)$, maioria com peso classificado como normal $(77,2 \%)$, foi encontrada numa minoria $(19,5 \%)$ dos adolescentes insatisfação com a imagem corporal. Tendo uma conclusão de que os dados do estudo, justificam a importância de tratar e prevenir os distúrbios de imagem corporal, para ajudar a alcançar os padrões alimentares mais saudáveis.

Embora vários estudos tenham realizados pesquisas com esses moldes, limitações importantes devem ser citadas como pesquisar apenas o sexo feminino como foi o caso de Bandeira et al. (2016), Oliveira (2017) e Alves et al. (2017), estudos como Guimarães et al. (2020) evidenciam em homens entre 15 e 24 anos, $75,1 \%$ de insatisfação com a imagem corporal o que sugere que a preocupação para esse distúrbio não dever ser direcionado apenas um sexo.

A não utilização de instrumentos adicionais para a mesma variável como Bandeira et al. (2016), Oliveira (2017) e Ribeiro-Silva et al. (2017) utilizaram o Questionário de Imagem Corporal (QIC), e Alves et al. (2017) e Guimarães et al. (2020) utilizaram a Escala de Figuras de Silhuetas (EFS) como únicas saídas para identificar a Insatisfação com a Imagem Corporal (IIC), o que poderia aumentar a assertividade da variável, caso fosse utilizadas as duas juntas.

Para a variável relacionado ao estado nutricional ou a condição corporal os estudos utilizaram o Índice de Massa Corporal (IMC) o que é um instrumento sugerido e de fácil manuseio para obtenção das medidas de estatura e massa corporal apesar de não fornecer informações precisas sobre a composição corporal, incluindo dessa forma como uma limitação do estudo (BANDEIRA et al., 2016). A natureza transversal dos estudos também é uma limitação que deve ser mencionada, pois acaba não considerando a sequência temporal dos eventos, e assim não determinando a causalidade, contudo estudos longitudinais podem fornecer um melhor entendimento para este tema.

\section{CONCLUSÕES}

Os resultados obtidos apontam maiores índices de insatisfação corporal em adolescentes do sexo feminino, com maior ocorrência da insatisfação por excesso de peso, quanto ao sexo masculino a insatisfação corporal teve sua maior ocorrência relacionada ao desejo de ganho de peso. O presente estudo se torna relevante pela relevância da temática fazendo com que os profissionais criem estratégias, projetos e intervenções para tratar deste tema com os alunos, gerando informação e saúde.

Apresenta-se como limitações o fato que a maioria das pesquisas terem sido realizada apenas com o sexo feminino e a não utilização de instrumentos adicionais para a mesma variável, dessa forma impossibilitando um resultado que inclua todos os adolescentes escolares. Haja vista que a autoimagem corporal pode trazer sérios prejuízos para o adolescente, dentre eles distúrbios de ordem mental, torna-se necessário uma reformulação ou criação de políticas de saúde e educação voltadas para a importância da realização de atividade física regular, hábitos saudáveis e para o psicológico dos adolescentes de forma que não sejam tão influenciados pelos meios de comunicação em massa. 


\section{REFERÊNCIAS}

AINETT, W. S. O.. Fatores associados à insatisfação com a imagem corporal em estudantes de nutrição. Revista Brasileira de Obesidade, nutrição e Emagrecimento, São Paulo, v.11, n.62, p.1-11, 2017.

ALVES, F. R.; BEZERRA, F. É. L.; SOUZA, E. A.; TEIXEIRA, F. A. A.. Imagem corporal e fatores associados em meninas de escolas militares. Revista Brasileira em Promoção da Saúde, v.30, n.4, p.1-7, 2017. DOI:

http://dx.doi.org/10.5020/18061230.2017.6082

BANDEIRA, Y. E. R.; MENDES, A. L. R. F.; CAVALCANTE, A. C. M.; ARRUDA, S. P. M.. Avaliação da imagem corporal de estudantes do curso de Nutrição de um centro universitário particular de Fortaleza. Revista Brasileira de Psiquiatria, Fortaleza, v.2, n.65, p.1-6, 2016

GUIMARÃES, B. E. B.. O consumo excessivo de álcool e a insatisfação com a imagem corporal por adolescentes e jovens de um município baiano, Brasil. Cadernos de Saúde Pública, v.36, n.1, p.1-15, 2020. DOI: http://dx.doi.org/10.1590/0102-311x044919

IEPSEN, A. M.; SILVA, M. C.. Prevalência e fatores associados à insatisfação com a imagem corporal de adolescentes de escolas do Ensino Médio da zona rural da região sul do Rio Grande do Sul, 2012. Epidemiologia e Serviços de Saúde, v.23, n.2, p.317-325, 2014. DOI: http://dx.doi.org/10.5123/s1679-49742014000200013

LIMA, F. É. B.; DRUMMOND, A. C.; BIGAI, D. C.; PELLEGRINOTTI, Í. L.; LIMA, W. F.; LIMA, S. B. S.; LIMA, F. B.. Insatisfação corporal e percepção da imagem corporal em adolescentes de Piraju/SP. Revista Biomotriz, Piraju, v.12, n.1, p.1-18, 2018.

MARTINI, M. C. S.; ASSUMPÇÃO, D.; BARROS, M. B. A.; BARROS FILHO, A. A.. Insatisfação com o peso corporal e estado nutricional de adolescentes: estudo de base populacional no município de Campinas, São Paulo, Brasil: estudo de base populacional no município de Campinas, São Paulo, Brasil. Ciência \& Saúde Coletiva, v.25, n.3, p.967-975,
2020. DOI: http://dx.doi.org/10.1590/1413$\underline{81232020253.17992018}$

MARTINS, C. R.; PETROSKI, E. L.. Insatisfação com a imagem corporal em adolescentes do sexo feminino de uma cidade de pequeno porte: prevalência e correlações: prevalência e correlações. Motricidade, v.11, n.2, p.1-13, 2015. DOI: http://dx.doi.org/10.6063/motricidade.3670

OLIVEIRA, D. S.. Percepção de adolescentes de uma escola municipal acerca da autoimagem corporal. Revista Sociedade Brasileira de Clínica Médica, Guanambi, v.2, n.15, p.1-6, 2017.

PETROSKI, E. L.. Motivos e prevalência de insatisfação com a imagem corporal em adolescentes. Revista de Ciência e Saúde Coletiva, Londrina, v.4, n.17, p.1-7, 2012.

RIBEIRO-SILVA, R. C.; FIACCONE, R. L.; CONCEIÇÃOMACHADO, E. P.; RUIZ, A. S.; BARRETO, M. L.; SANTANA, M. L. P.. Body image dissatisfaction and dietary patterns according to nutritional status in adolescents. Jornal de Pediatria, v.94, n.2, p.155-161, 2018. DOI: http://dx.doi.org/10.1016/j.jped.2017.05.005

SANTOS, J. F. S.; OLIVEIRA, P.; CAMPOS, A. A.; PEREIRA, E. O.; SOUSA, E. A.. Relação entre Insatisfação Corporal e Atividade Física em Adolescentes da Zona Urbana de Irati-PR. Revista da Educação Física, v.25, n.2, p.1-9, 2014. DOI: http://dx.doi.org/10.4025/reveducfis.v25i2.21991

TABORDA, J. A.; GONÇALVES, H. C. B.; SILVA, J. D. I.; SOARES, P. P. D.; VIEIRA, R. V.; RIBEIRO, V. COZAC, E. E..

Consequências da gravidez na adolescência para as meninas considerando-se as diferenças socioeconômicas entre elas. Cadernos Saúde Coletiva, v.22, n.1, p.16-24, 2014. DOI: http://dx.doi.org/10.1590/1414-462x201400010004

VIANA, D. S. O.. Análise nutricional das dietas da moda. Monografia (Bacharelado em Nutrição) - Centro Universitário de Brasília, Brasília, 2014.

A CBPC - Companhia Brasileira de Produção Científica (CNPJ: 11.221.422/0001-03) detém os direitos materiais desta publicação. Os direitos referem-se à publicação do trabalho em qualquer parte do mundo, incluindo os diritos às renovã̃es, expansões e disseminac̃os da contribuição, bem como outros direitos subsidiérios. Todos os trabalhos publicados eletronicamente poderáo posteriormente ser publicados em coletâneas impressas sob coordenação da Sapientiae Publishing, da Companhia Brasileira de Produção Científica e seus parceiros autorizados. Os (as) autores (as) preservam os direitos autorais, mas não têm permissão para a publicação da contribuição em outro meio, impresso ou digital, em português ou em tradução. 